\title{
Mineraçãa
}

\section{Concentração por flotação da apatita proveniente de rochas de filiação carbonatítica}

\author{
Concentration by apatite flotation originating \\ from carbonatically affiliated rocks
}

Paulo Renato Perdigão Paiva

Instituto de Geociências

Universidade de Brasília

Campus Universitário Darcy Ribeiro,

Brasília, DF, Brasil

paulorenato.paiva@gmail.com

Marisa Bezerra de Mello Monte

Laboratório de Química de Superfície,

Coordenação de Processos Minerais

CETEM - Rio de Janeiro, Brasil

mmonte@cetem.gov.br

José Carlos Gaspar

Instituto de Geociências

Universidade de Brasília

Campus Universitário Darcy Ribeiro,

Brasília, DF, Brasil

gasp@unb.br

\section{Resumo}

Esse estudo de processamento busca o aprimoramento das operações de flotação direta e de flotação reversa da apatita proveniente de rochas de filiação carbonatítica, objetivando obter um concentrado de apatita na especificação da indústria de ácido fosfórico. A flotação direta da apatita foi realizada em duas etapas de flotação, rougher e cleaner. O objetivo da flotação direta foi gerar um produto para alimentar o circuito de flotação reversa da apatita. A flotação reversa foi realizada em três etapas (rougher, cleaner e recleaner) e em pH igual a 5,0, utilizando o ácido fosfórico $\left(\mathrm{H}_{3} \mathrm{PO}_{4}\right)$ como depressor da apatita. Em linhas gerais, os resultados dos ensaios de flotação reversa evidenciaram a recuperação da apatita, o que demonstra que os reagentes específicos utilizados favoreceram a seletividade do sistema. Foi possível obter um concentrado de apatita com $38,11 \%$ de $\mathrm{P}_{2} \mathrm{O}_{5}, 50,98 \%$ de $\mathrm{CaO}, 1,07 \%$ de $\mathrm{Fe}_{2} \mathrm{O}_{3}, 0,67 \%$ de $\mathrm{MgO}$ e razão $\mathrm{CaO} / \mathrm{P}_{2} \mathrm{O}_{5}$ de 1,34 .

Palavras-chave: Flotação, minério sílico-carbonatado, carbonatos, apatita, ácido fosfórico.

\section{Abstract}

The current processing study seeks to improve operations for the direct and reverse flotation of apatite originating from carbonatically-affiliated rocks to obtain an apatite concentrate in accordance with the phosphoric acid industry specifications. The direct flotation of apatite was carried out in two flotation phases, rougher and cleaner. The aim of direct flotation was to generate a product to feed the reverse flotation circuit of apatite. Reverse flotation was performed in three stages (rougher, cleaner and recleaner) and at $p H$ 5.0, using phosphoric acid $\left(\mathrm{H}_{3} \mathrm{PO}_{4}\right)$ as an apatite depressor. Summing up, the results of reverse flotation tests pointed out apatite recovery, which shows that the specific reagents used favored system selectivity. It was possible to obtain an apatite concentrate with $38.11 \%$ of $\mathrm{P}_{2} \mathrm{O}_{5}, 50.98 \%$ of $\mathrm{CaO}, 1.07 \%$ of $\mathrm{Fe}_{2} \mathrm{O}_{3}, 0.67 \%$ of $\mathrm{MgO}$ and ratio $\mathrm{CaO} / \mathrm{P}_{2} \mathrm{O}_{5}$ of 1.34

Keywords: Flotation, silicon-carbonate ore, carbonates, apatite, phosphoric acid.

\section{Introdução}

As rochas de filiação carbonatítica formam, através de intemperismo químico, o minério sílico-carbonatado. Nos afloramentos intemperizados, o minério sílico-carbonatado ocorre sob a forma de rocha semi-alterada a muito alterada, mas ainda conservando a estrutura original (Le
Maitre, 2002; Lee et al., 2004; Krasnova et al., 2004 a,b; Abouzeid, 2008). De acordo com Ribeiro (2008), o minério sílico-carbonatado pode ser subdividido em função da litologia que lhe deu origem em minério foscorítico e minério flogopitítico.

A dificuldade encontrada na con- 
centração do minério sílico-carbonatado por flotação está intimamente ligada à composição química da apatita, que varia, significativamente, nas diferentes rochas portadoras de mineralização apatítica (Pearse, 2005; Lee et al., 2004).

A liberação do mineral é outro fator que afeta fortemente a seletividade e a concentração da apatita (Al-Wakeel et al., 2009), contida no minério sílico-carbonatado, pela diversidade de minerais de ganga associados. No caso do minério sílicocarbonatado, as operações de cominuição acarretam, ainda, uma transformação significativa na textura dessas partículas, afetando o seu desempenho no processo de flotação. Mesmo no caso de partículas de apatita liberadas, a eficiência do processo de flotação poderá ser dependente das reações de dissolução do sistema apatita/ carbonatos, modificando as características físico-químicas de superfície dos minerais, que constituem a alimentação da flotação (Amankonah \& Somasundaran, 1985; Guimarães et al., 2005).

\section{Experimental}

\section{Materiais}

A amostra run of mine (ROM) estudada era constituída, essencialmente, pelo minério foscorítico contendo $7,58 \%$ de $\mathrm{P}_{2} \mathrm{O}_{5}, 29,21 \%$ de $\mathrm{CaO}$, $10,05 \%, \mathrm{MgO}, 6,80 \%$ de $\mathrm{SiO}_{2}$ e 17,50 de $\mathrm{Fe}_{2} \mathrm{O}_{3}$.

A amostra ROM foi britada e clas-
Devido à baixa seletividade dos processos de flotação de apatita, em relação aos carbonatos e ao fato de os depósitos de apatita terem uma mineralogia extremamente complexa, tendo contaminantes que influenciam no rendimento de fósforo nas usinas de beneficiamento (Abouseid, 2008; Segul et al., 2006; Zafar et al., 2006; Blazy \& Bouhaouss, 2005; Zhong et al. 1993; Mishra, 1982), a flotação de carbonatos com depressão de apatita, utilizando ácido fosfórico, é uma alternativa que vem sendo estudada, principalmente, quando o minério contém baixo teor de apatita e uma concentração expressiva de dolomita e calcita.

O processo de flotação de carbonatos foi estudado por Abramov et al. (1993) no beneficiamento de um minério fosfático proveniente da Turquia. No processo descrito pelos autores, no primeiro estágio de flotação, foram recuperados apatita e os carbonatos, em $\mathrm{pH}=8,0$. Em outra etapa, a flotação foi conduzida com o oleato de sódio e a depressão do fosfato foi obtida na faixa de $\mathrm{pH}$ situada entre 5,0 e 6,0, utilizando-se $\mathrm{H}_{3} \mathrm{PO}_{4}$. De acordo com os pesquisadores, na flotação conjunta dos minerais de apatita e calcita realizada na faixa alcalina de $\mathrm{pH}$, os íons oleato adsorvem-se quimicamente na superfície desses minerais e os íons $\mathrm{PO}_{4}^{3-}$ e F-, provenientes da solubilização da apatita, irão competir com os íons oleato pelos sítios de cálcio na superfície da apatita e da calcita. Quando o $\mathrm{H}_{3} \mathrm{PO}_{4}$ é adicionado, na etapa de flotação reversa, a concentração do íon $\mathrm{PO}_{4}$ - aumenta, interferindo no equilíbrio, visto que a concentração desse íon torna-se maior que a concentração do íon oleato. Por outro lado, a calcita não sofre a influência desse equilíbrio e, conseqüentemente, do aumento da concentração do íon $\mathrm{PO}_{4}{ }^{3-}$, sendo recuperada na espuma de flotação.

Dentro desse contexto, esse trabalho buscou desenvolver uma rota alternativa no processamento do minério sílicocarbonatado, utilizando o ácido fosfórico como depressor da apatita presente no minério sílico-carbonatado.

\section{Análises de imagens por microscopia eletrônica de varredura}

As análises de imagens por Microscopia Eletrônica de Varredura (MEV) foram efetuadas num equipamento LEO S440, equipado com detector de elétrons retroespalhados, acoplado a um espectrômetro de dispersão de energia de raios $\mathrm{X}$ (EDS), Oxford Link L300, com detector de SiLi Pentfet e janela ultrafina ATW2, com resolução de $133 \mathrm{eV}$ a $5,9 \mathrm{keV}$. Essa técnica foi empregada para se obterem as

\section{Ensaios de flotação}

O processamento das amostras, para os ensaios de flotação direta da apatita, consistiu das operações unitárias de moagem $\left(\mathrm{P}_{80}=250 \mu \mathrm{m}\right)$, separação magnética (1000 Gauss), atrição em pH natural da polpa e deslamagem (fração $<37 \mu \mathrm{m}$ ), respectivamente.

Para a flotação reversa da apatita sificada em peneira SWECO, revestida com tela de abertura de 2,0 $\mathrm{mm}$.

Os coletores utilizados foram o sulfossuccinamato diluído a $1 \% \mathrm{p} / \mathrm{v}$ e o hidrocol saponificado com hidróxido de sódio a $5 \% \mathrm{p} / \mathrm{v}$. Os depressores utilizados foram o amido gelatinizado com hidróxido de sódio a 4\% p/v, o metasilicato de sódio, que atua como depressor de filossilicatos e dispersante para as lamas, e o acido fosfórico $\left(\mathrm{H}_{3} \mathrm{PO}_{4}\right) \mathrm{com}$ 98\% de pureza da VETEC Company. Para ajuste do $\mathrm{pH}$, foram utilizadas soluções de $\mathrm{NaOH}$ e $\mathrm{H}_{2} \mathrm{SO}_{4}$ a $10 \%$ p/v. imagens para o estudo de liberação.

Para as análises de imagens, as alíquotas foram classificadas a úmido nas peneiras de $297 \mu \mathrm{m}, 210 \mu \mathrm{m}, 149 \mu \mathrm{m}$, $103 \mu \mathrm{m}, 74 \mu \mathrm{m}, 53 \mu \mathrm{m}, 44 \mu \mathrm{m}, 37 \mu \mathrm{m}$ e $20 \mu \mathrm{m}$ e, de cada fração, foi preparada uma secção polida para geração das imagens por MEV. A face polida foi recoberta com carbono condutor.

As imagens geradas por MEV foram

(flotação direta dos carbonatos), o processamento das amostras consistiu na remoagem, em $\mathrm{P}_{80}$ de $75 \mu \mathrm{m}$ e deslamagem em $20 \mu \mathrm{m}$, do concentrado cleaner obtido na etapa de flotação direta da apatita.

Os ensaios de flotação foram realizados em célula de laboratório Denver subaerada, modelo D12, utilizando-se uma transferidas para uma estação de trabalho Linux, para serem processadas utilizando um software especializado para análise de liberação (MMIA - Mineral Metallurgical Image Analysis) de King e Schneider (1993). A simulação de liberação é baseada em um modelo de balanço populacional, que permite que o cálculo seja feito independentemente das características do minério (King, 1990; Schneider, 1995).

cuba nominal de $3 \mathrm{~L}$, amostras de 1,000 g, velocidade de agitação de $1200 \mathrm{rpm}$ para a etapa de condicionamento e $1400 \mathrm{rpm}$ nas etapas de flotação rougher e cleaner.

Os processos de flotação foram realizados em dois estágios. No primeiro estágio, denominado de flotação direta da apatita, utilizando duas etapas de flotação 
(rougher e cleaner), foram recuperados a apatita e os carbonatos, em $\mathrm{pH}=8,0$. Os reagentes utilizados foram os depressores metassilicato de sódio $(1,0 \mathrm{mg} / \mathrm{g})$, amido $(0,5 \mathrm{mg} / \mathrm{g})$ e o coletor sulfossuccinamato (1,0 mg/g). Os tempos de condicionamento foram de $5 \mathrm{~min}$ para os depressores e de 1

\section{Resultados}

\section{Estudo de liberação}

A Figura 1 mostra o espectro de liberação da apatita contida no minério foscorítico. Observa-se que a liberação da ganga atinge valores acima de 55\% das partículas, para todas as classes de tamanho. Por outro lado, a apatita $100 \%$ liberada não atinge $12 \%$ das partículas. Uma particularidade que ocorre com a liberação é que, após um discreto aumento

Figura 1

Espectro de liberação da apatita, condicional por tamanho.

Figura 2

Detalhes das inclusões finas nas granulações mais grossas.

Figura 3

Curva de projeção de recuperação global da apatita versus descarte de massa, calculada a partir dos dados de liberação min para o coletor.

No segundo estágio, denominado de flotação direta dos carbonatos ou flotação reversa da apatita, foram recuperados os carbonatos com conseqüente depressão da apatita, em $\mathrm{pH}=5,0$, utilizando-se três etapas de flotação (rougher, cleaner e recleaner). $\mathrm{O} \mathrm{H}_{3} \mathrm{PO}_{4}$ foi utilizado como depressor da apatita. A flotação foi realizada com o coletor hidrocol $(0,5 \mathrm{mg} / \mathrm{g})$ e o espumante metil isobutil carbinol (MIBIC, $0,10 \mathrm{mg} / \mathrm{g})$. Os tempos de condicionamento foram de $3 \mathrm{~min}$ para o depressor e de 1 min para o coletor e o espumante. entre as granulações de $297 \mu \mathrm{m}$ e $74 \mu \mathrm{m}$, a liberação volta a diminuir nas granulações mais finas (44 $\mu \mathrm{m}$ a $20 \mu \mathrm{m})$. Essa diminuição pode estar relacionada com a presença de finas inclusões de minerais de ganga na apatita de granulações mais grossas, como mostra a imagem da Figura 2 (gerada pelo MEV), e estas inclusões deslocariam a liberação da apatita em direção a uma classe de menor teor.

As partículas de ganga liberada ou contendo pouca apatita, que correspondem a até $70 \%$ da massa do minério, podem ser removidas para o rejeito, sem implicar perdas significantes de apatita, como mostra a curva de separação ideal entre as populações de partículas (Figura 3).
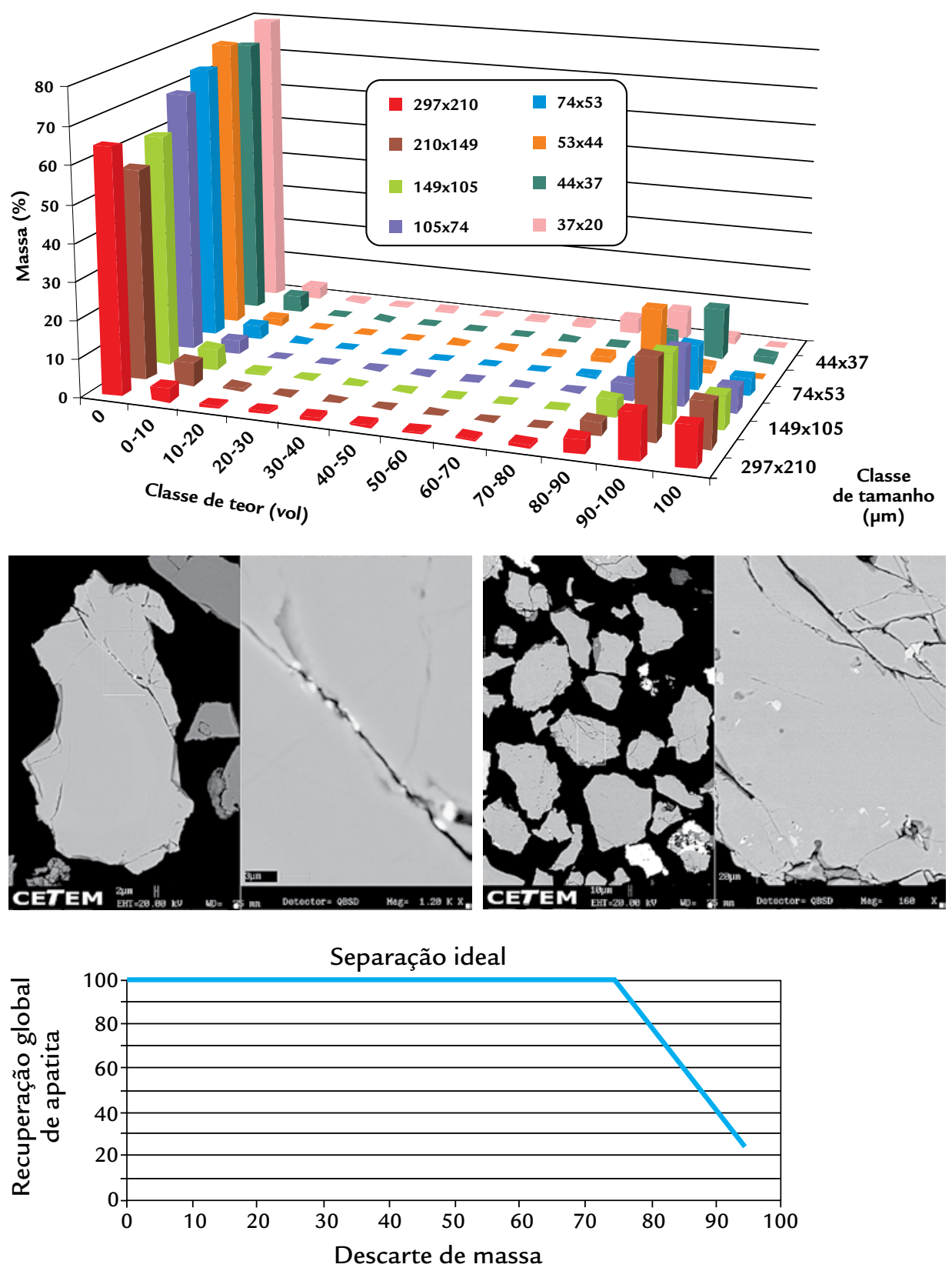


\section{Ensaios de flotação}

A Tabela 1 apresenta as recuperações em massa, metalúrgica, bem como os percentuais dos elementos principais contidos nos produtos obtidos para as diferentes operações adotadas. Conforme os resultados, pode-se observar que, após a separação magnética, deslamagem e atrição, ocorreram mudanças significativas nos teores de $\mathrm{P}_{2} \mathrm{O}_{5}, \mathrm{CaO}$ e $\mathrm{MgO}$. Observa-se um incremento no teor de $\mathrm{P}_{2} \mathrm{O}_{5}$ de $7,58 \%$ para $10,17 \%$. A separação magnética e a deslamagem removeram 42,03\% do $\mathrm{MgO}, 54,73 \%$ de $\mathrm{SiO}_{2}$ e $74,11 \%$ de $\mathrm{Fe}_{2} \mathrm{O}_{3}$ contido no minério ROM. O material obtido, após a deslamagem, consistiu da alimentação do circuito de flotação direta da apatita.

A Figura 4 mostra que a flotação direta da apatita atingiu um teor máximo, no concentrado cleaner, de $\mathrm{P}_{2} \mathrm{O}_{5}$ de $17,90 \%$ com uma recuperação no circuito de flotação de $85,87 \%$. Observa-se também que a flotação removeu $21,70 \%$ do $\mathrm{MgO}, 35,70 \%$ de $\mathrm{SiO}_{2}$ e $25,11 \%$ do $\mathrm{Fe}_{2} \mathrm{O}_{3}$.

O resultado obtido no ensaio de

\begin{tabular}{|c|c|c|c|c|c|c|c|}
\hline \multirow{2}{*}{$\begin{array}{l}\text { Operações } \\
\text { unitárias }\end{array}$} & \multirow{2}{*}{ Produtos } & \multirow{2}{*}{$\begin{array}{l}\text { Peso } \\
(\%)\end{array}$} & \multicolumn{5}{|c|}{ Recuperação (\%) } \\
\hline & & & $\mathbf{P}_{2} \mathrm{O}_{5}$ & $\mathrm{CaO}$ & $\mathrm{MgO}$ & $\mathrm{Fe}_{2} \mathrm{O}_{3}$ & $\mathrm{SiO}_{2}$ \\
\hline \multirow{2}{*}{$\begin{array}{l}\text { Separação } \\
\text { magnética }\end{array}$} & Produto não magnético & 79.80 & 92.81 & 91.15 & 85.95 & 36.86 & 86.90 \\
\hline & Produto magnético & 20.20 & 7.19 & 8.85 & 14.05 & 63.14 & 13.10 \\
\hline \multirow{2}{*}{$\begin{array}{l}\text { Remoção da } \\
\text { fração }<37 \mu \mathrm{m}\end{array}$} & Finos & 19.00 & 11.98 & 16.52 & 27.98 & 10.97 & 41.63 \\
\hline & Alimentação do circuito de flotação & 60.80 & 80.83 & 74.63 & 57.97 & 25.89 & 45.27 \\
\hline \multirow{2}{*}{$\begin{array}{l}\text { Operações } \\
\text { unitárias }\end{array}$} & \multirow{2}{*}{\multicolumn{2}{|c|}{ Produtos }} & \multicolumn{5}{|c|}{ Teor $(\%)$} \\
\hline & & & $\mathbf{P}_{2} \mathrm{O}_{5}$ & $\mathrm{CaO}$ & $\mathrm{MgO}$ & $\mathrm{Fe}_{2} \mathrm{O}_{3}$ & $\mathrm{SiO}_{2}$ \\
\hline \multirow{2}{*}{$\begin{array}{l}\text { Separação } \\
\text { magnética }\end{array}$} & \multicolumn{2}{|l|}{ Produto não magnético } & 8.82 & 33.36 & 10.82 & 8.08 & 7.40 \\
\hline & \multicolumn{2}{|l|}{ Produto magnético } & 2.70 & 12.80 & 6.99 & 54.70 & 4.41 \\
\hline \multirow{2}{*}{$\begin{array}{c}\text { Remoção da } \\
\text { fração }<37 \mu \mathrm{m}\end{array}$} & \multicolumn{2}{|l|}{ Finos } & 4.78 & 25.40 & 14.80 & 10.10 & 14.90 \\
\hline & \multicolumn{2}{|l|}{ Alimentação do circuito de flotação } & 10.17 & 35.94 & 9.59 & 7.49 & 5.10 \\
\hline
\end{tabular}

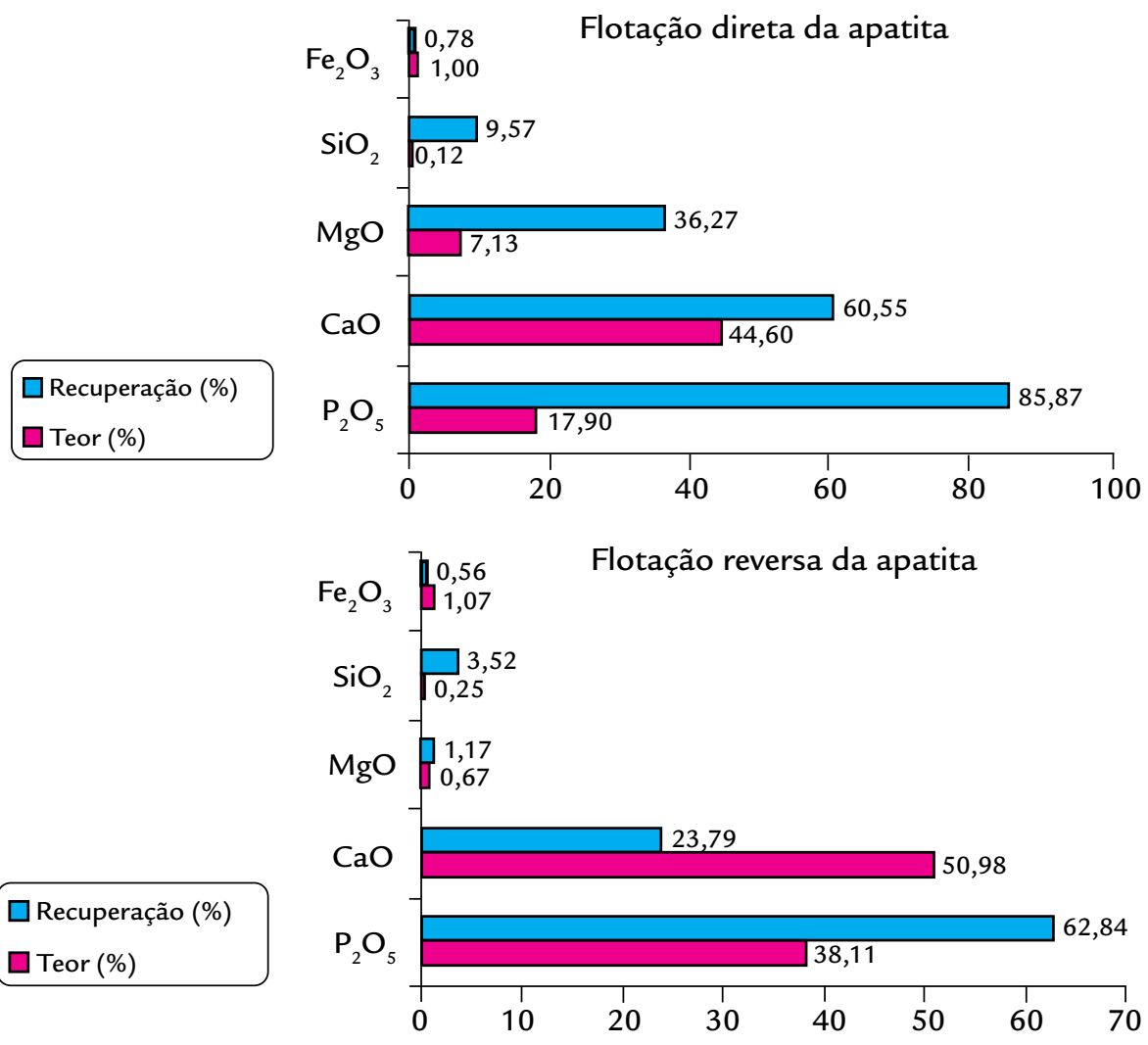

Tabela 1

Recuperações em massa, metalúrgica e teores obtidos nas diferentes operações unitárias.

Figura 4

Fluxograma apresentando os teores e as recuperações contidas no produto obtido na etapa de flotação direta da apatita.

Figura 5

Fluxograma apresentando os teores e as recuperações contidas no produto obtido na etapa de flotação reversa da apatita.

\section{Discussões}

As operações unitárias de moagem $\quad\left(\mathrm{P}_{80}=250 \mu \mathrm{m}\right)$, atrição em $\mathrm{pH}$ natural, separação magnética (1000 Gauss) e des- 
lamagem (fração < $37 \mu \mathrm{m}$ ), foram etapas essenciais no processo, uma vez que, normalmente, os ensaios de flotação requerem amostras com uma distribuição granulométrica controlada, já que o tamanho da partícula e a presença/ausência de lama são variáveis fundamentais no processo de flotação, com forte influência na cinética e na recuperação (Bravo et al., 2005; Pease et al., 2006).

A determinação da granulometria de moagem em $\mathrm{P}_{80}$ de 250 micrômetros foi realizada a partir do estudo de liberação (Figuras 1 e 3). Esse valor, relativamente alto, reduz as perdas na etapa de deslamagem e favorece a separação da ganga silicática, magnetitas e de grande parte dos carbonatos. Na Figura 1, fica claro que a concentração da apatita é uniforme para a granulação mais grossa $(297 \mu \mathrm{m}$ a $53 \mu \mathrm{m})$.

A remoagem do concentrado obtido na flotação direta da apatita teve como principal função gerar superfícies frescas nos minerais constituintes, basicamente, dolomita, calcita e apatita, portanto a liberação de apatita não é o objetivo primário da etapa de remoagem. A remoagem foi realizada com $\mathrm{P}_{80}$ de $75 \mu \mathrm{m}$, pois esse valor, aparentemente, foi mais adequado às condições de flotabilidade dos carbonatos.

\section{Conclusões}

As operações unitárias e a etapa de flotação direta foram essenciais ao processo, uma vez que elas favoreceram a remoção da ganga silicática, magnetitas e uma parte da ganga carbonática, contribuindo para que a flotação reversa alcance bom resultado.

A flotação direta da apatita, seguida da flotação reversa da apatita, apresentou uma recuperação em massa e uma recuperação de $\mathrm{P}_{2} \mathrm{O}_{5}$, no circuito de flotação, de $16,77 \%$ e $62,84 \%$, respectivamente.

\section{Referências bibliográficas}

As etapas de deslamagem realizadas, após a moagem do minério ROM e a remoagem do concentrado cleaner, foram utilizadas com o intuito de minimizar a aderência de partículas ultrafinas nas superfícies das partículas grossas (esse fenômeno é denominado de slime coating) e essa interação entre as partículas ultrafinas e grossas diminuiu a seletividade induzida pelo sistema de reagente empregado. Além disso, as gerações de partículas finas ou ultrafinas criam superfícies ativas de cátions metálicos $\left(\mathrm{Al}^{3+}, \mathrm{Fe}^{2+}, \mathrm{Fe}^{3+}, \mathrm{Mg}^{2+}, \mathrm{Mn}^{2+}\right.$, $\mathrm{Ca}^{2+}$ ), os quais funcionam como sítios para a adsorção específica do coletor (Johnston \& Leja, 1978). Entretanto, $\mathrm{o}_{2} \mathrm{O}_{5}$ presente nas lamas não pode ser considerado como perda, pois as referidas lamas podem ser submetidas ao processo de flotação em coluna, aumentando a recuperação da apatita presente nesse minério.

A baixa seletividade no processo de flotação direta da apatita (Figura 4) demonstra que, para o minério sílicocarbonatado, os reagentes específicos utilizados não favorecem a obtenção de um concentrado rico em apatita. Em linhas gerais, os resultados dos ensaios de flotação direta evidenciaram a recuperação conjunta dos carbonatos e da apatita. A associação da apatita com os carbonatos foi considerada um dos principais impedimentos para o aumento da seletividade do processo de flotação utilizado.

Acredita-se que a flotação reversa da apatita alcançou bons resultados (Figura 5), porque, em pH=5,0, a espécie do ácido fosfórico predominante em solução é o íon $\mathrm{H}_{2} \mathrm{PO}_{4}$ e esse íon é, provavelmente, responsável pela depressão da apatita. Esse íon $\left(\mathrm{H}_{2} \mathrm{PO}_{4}^{-}\right)$adsorve-se na superfície da apatita e forma pontes de hidrogênio com moléculas de água, tornando a superfície da apatita hidrofílica, como foi demonstrado por Louzada et al. (2008). Além disso, o processo de flotação reversa da apatita, em $\mathrm{pH}=5,0$, minimiza a dissolução/precipitação dos íons cálcio e magnésio na superfície da apatita, aumentando a eficiência do processo. Em circuitos industriais, concentrações de íons $\mathrm{Ca}^{2+}$ e $\mathrm{Mg}^{2+}$, na faixa entre 500 e 1000mg.l-1, respectivamente, não são incomuns (Giesekke \& Harris, 1994).

Destaca-se que os critérios de escolha dos reagentes foram baseados em dados oferecidos pela literatura especializada (Johnston \& Leja, 1978; Aquino et al., 1985; Abramov et al., 1993; Guimarães et al., 2005; Louzada et al., 2008) e pela experimentação intensiva.
Apresentou, ainda, um concentrado fosfático com a seguinte especificação: $38,11 \%$ de $\mathrm{P}_{2} \mathrm{O}_{5}, 50,98 \%$ de $\mathrm{CaO}, 1,07 \%$ de $\mathrm{Fe}_{2} \mathrm{O}_{3}$, $0,67 \%$ de $\mathrm{MgO}$ e razão $\mathrm{CaO} / \mathrm{P}_{2} \mathrm{O}_{5}$ de 1,34 .

Considerando os resultados obtidos para o circuito de flotação, pode-se afirmar que a metodologia aplicada nesse estudo é pertinente, ou seja, a flotação direta da apatita, seguida da flotação direta de carbonatos, pode ser considerada uma rota viável para a obtenção de um concentrado de apatita dentro da especificação da indústria de ácido fosfórico.

A metodologia aplicada nesse estudo pode ser estendida a outros depósitos de rocha fosfática e minérios relacionados a complexos carbonatíticos em que os teores de fósforo são economicamente compatíveis, mas a presença da dolomita e/ou calcita e de suas associações não permite seu processamento e aplicação, sendo consideradas como estéreis.

ABOUZEID, A.Z.M. Physical and thermal treatment of phosphate ores - an overview. International Journal Mineral Processing, v. 85, p. 59-84, 2008.

ABRAMOV, A. A., ABRAMOV, AL., ONAL, G., ATAK, S., CELIK, M. S. Mechanism of reverse flotation of calcareous phosphate ores. In: EL-SHALL, H., MOUDGIL, B. M., WIEGEL, R. (Eds.): Beneficiation of phosphate. Theory and practice. Littleton, Colorado: 1993. Cap.29, p.281-288.

AQUINO, J. A., FURTADO, J. R. V., REIS JUNIOR, J. B. Concentration of phosphate ore with siliceous-carbonated gangue via reverse flotation. In: LATIM-AMERICAN CONGRESS ON FROTH FLOTATION, 2. Proceedings... Concepcíon-Chile, august, p. 185-200, 1985.

AL-WAKEEL, M. I., LIN, C. L., MILLER, J.D. Significant of liberation characteristics in the fatty acid flotation. Minerals Engineering, v. 22, p. 244-253, 2009.

AMANKONAH J., SOMASUNDARAN, P. Effects of dissolved mineral on the electrokinetic behavior of calcite and apatite. Colloids and Surfaces, v. 15, p. 335-353, 1985.

BLAZY, P., BOUHAOUSS, A. Removal of organic matter in Moroccan Youssoufia phosphate by flash calcinations. Minerals \& Metallurgical Processing, v. 22, n. 2, p.107-115, 2005. 
BORN, H., KAHN, H. Caracterização geológica e mineralógica voltada ao aproveitamento de jazimento fosfáticos. In: ENCONTRO NACIONAL DE ROCHAS FOSFÁTICAS - IBRAFOS, 5. São Paulo: 1990.

BRAVO, S.V.C., TOREM, M.L., MONTE, M. B. M., DUTRA, A. J. B., TONDO, L.A. The influence of particle size and collector on the flotation of a very low grade auriferous ore. Minerals Engineering, v. 18, p. 459-461, 2005.

GIESEKKE, E. W., HARRIS, P. J. The role of polyoxyethylene alkyl ethers in apatite flotation at Foskor, Phalaborwa (South Africa). Minerals Engineering, v. 7 1, p. 1345-1361, 1994.

GUIMARAES, R. C., ARAUJO, A. C., PERES, A. E. C. Reagents in igneous phosphate ores flotation. Minerals Engineering, v.18, p. 199-204, 2005.

JOHNSTON, D. J., LEJA, J. Flotation behaviour of calcium phosphates and carbonates in orthophosphate solution. Transactions of the Institution of Mining and Metallurgy, London, v.87, Section C, p. 237-242, 1978.

LOUZADA, J. C. G., AQUINO, J. A., OLIVEIRA, J. F. Selective flotation of calcite from apatite by using phosphoric acid and citric as depressants. In: Beneficiation of phosphates: technology advance and adoption. Published by: Society for Mining, Metallurgy \& Exploration (SME). Littleton, Colorado, USA. Section 7, p. 305-310, 2008. ISBN-13: 978-0-87335-319-9.

KRASNOVA, N. I., BALAGANSKAYA, E. G., GARCIA, D. Kovdor-classic phoscorites and carbonatites. In: WALL, F., ZAITZEV, A. N. (Eds.). Phoscorites and carbonatites from mantle to mine: the key example of the Kola Alcaline Province, 4. Mineralogical Society of Great Britain \& Ireland. London Press, 2004a. p.99-132.

KRASNOVA, N. I., PETROV, T. G., BALAGANSKAYA, E. G., GARCIA, D., MOUTTE, J., ZAITSEV A. N., WALL, F. Introduction to phoscorites: occurrence, composition, nomenclature and petrogenesis. In: WALL, F., ZAITZEV, A.N. (Eds). Phoscorites and carbonatites from mantle to mine: the key example of the Kola Alcaline Province, 2. Mineralogical Society of Great Britain \& Ireland. London Press, 2004b. p. 45-74.

KING, R. P. Calculation of the Liberation Spectrum in Products Produced. In: COUNTINUOS MILLING CIRCUITS - EUROPEAN SYMPOSIUM ON COMMINUTION, 7. Proceedings... Ljubljana: 1990. 429p.

KING, R. P., SCHNEIDER, C. L. An effective SEM-based image analysis for quantitative mineralogy. KONA, v.11, p. 165-177, 1993.

LEE, M. J., GARCIA, D., MOUTTE, J., WILlIAMS C. T., WALL, F. Carbonatites and phoscorites from de Sokly complex, Finland. Introduction to phoscorites: occurrence, composition, nomenclature and petrogenesis. In: WALL, F., ZAITZEV, A. N. (Eds.). Phoscorites and carbonatites from mantle to mine: the key example of the Kola Alcaline Province, 5. Mineralogical Society of Great Britain \& Ireland. London Press, 2004. p. 133-162.

LE MAITRE, R.W. Igneosu rocks. A classification and glossary terms. Cambridge University press. Cambridge, UK: 2002. 236p.

MISHRA, S. K. Electrokinetic properties and flotation behavior of apatite and calcite in the presence of sodium oleato metasilicate. International Journal Mineral Processing, v.9, p. 59-73, 1982.

PEASE, J. D., CURRY, D. C., YOUNG, M. F. Designing flotation circuits for fines recovery. Mineral Processing, v. 19, p. 831-840, 2006.

PEARSE, M. J. An overview of the use of chemical reagents in mineral processing. Minerals Engineering, v.18, p.139-149, 2005.

RIBEIRO, C. C. Geologia, geometalurgia, controles e gênese dos depósitos de fósforo, terras raras e titânio do complexo carbonatítico catalão I, GO. Universidade de Brasília, UNB, Brasil, 2008. (Tese de Doutorado em Geologia).

SCHNEIDER, C. L. Measurement and calculation of liberation in continuous milling circuits. Departamento de Engenharia Metalúrgica, Universidade de Utah, 1995. 356 p. (Tese de Doutorado).

SENGUL, H., OZER, A. K., GULABOGLU, M. S. Beneficiation of Mardin-Mazidagi (Turkey) calcareous phosphate rock using dilute acetic acid solutions. Chemical Engineering Journal, v. 122, p.135-140, 2006.

ZAFAR, I. Z., ANWAR, M. M., PRITCHARD, D. W. Selective leaching of calcareous phosphate rock in formic acid: optimisation of operating condictions. Minerals Engineering, v. 19, p.1459-1461, 2006.

ZOHNG, T. V., VASUDEVAN, A. N., SUMASANDARAN, P. Flotability of apatites of different type and origin: role of surface area and porosity. International Journal of Mineral Processing, v. 38, p.177-188, 1993. 